\title{
Noise-induced transition in a quantum system
}

\author{
Pulak Kumar Ghosh, Debashis Barik and Deb Shankar Ray* \\ Indian Association for the Cultivation of Science, Jadavpur, Kolkata 700 032, India
}

\begin{abstract}
We examine the noise-induced transition in a fluctuating bistable potential of a driven quantum system in thermal equilibrium. Making use of a Wigner canonical thermal distribution for description of the statistical properties of the thermal bath, we explore the generic effects of quantization like vacuum field fluctuation and tunneling in the characteristic stationary probability distribution functions undergoing transition from unimodal to bimodal nature and in signal-to-noise ratio characterizing the co-operative effect among the noise processes and the weak periodic signal.
\end{abstract}

PACS numbers: 05.40.+j, 02.50.-r

\section{INTRODUCTION}

The role of the noise in nonlinear dissipative systems has been an intriguing issue over the last two decades 1.2.3.4.5,6.7.8.9,10,11,12,13,14,15. Because of their potential applications, various noise-induced processes in zero dimensional and spatially extended systems, such as, stochastic resonance ${ }^{4.5 .6 .7}$, coherence resonance ${ }^{16}$, thermal ratchet ${ }^{17.18 .19}$, noise-induced front propagation ${ }^{8}$, noise-induced patten formation ${ }^{9 \cdot 10.11 .12 .13 .14}$ etc have been the subject of intense investigation in widely different areas of physical, chemical and biological sciences. An important early endeavour in this direction is the noise-induced transition 1.15 which plays a key role in understanding of the transition of unimodular character of stationary probability distribution to bimodal one as one varies the strength of external multiplicative noise in a bistable potential ${ }^{1.24 .25}$. The theory has been further advanced ${ }^{24.25}$ in several directions to include the aspects of localization, the effect of correlation between the noise processes, the cooperativity between the noise processes and the signal and the associated transient characteristics. For example, it had been shown earlier ${ }^{24}$ that a system in a bistable fluctuating potential driven by a very slow modulation or static tilting in presence of an additive noise exhibits localization in one of the wells. The stationary probability distribution is markedly sensitive to the variation of the correlation between the additive and multiplicative noises. Keeping in view of these observations on noise-induced transition in classical systems it is imperative that quantization ${ }^{20,21.22 .23}$ is likely to affect these features since tunneling and other generic quantum effects start playing significant role in a wider context. As prototypical experimental systems ${ }^{26}$ it is now possible possible to confine electrons in semiconductors, for example, in a quantum dot coupled to an environment via point contacts using nanolithographic techniques to explore in detail the quantum transport in relation to localization and tunneling.

The object of the present paper is to study noise-induced transition in a fluctuating bistable potential in a quantum system driven by a sinusoidal slowly varying field in presence of additive thermal noise of the heat bath. Our aim here is to explore the characteristics of the stationary probability distribution functions to understand the nature of noise-induced transition in presence of generic quantum effects like tunneling and vacuum fluctuations. In addition we also look for how these changes in the stationary probability distribution functions are reflected in the nonlinear co-operative effect between the noises and the signal.

\section{QUANTUM STOCHASTIC DYNAMICS OF A DRIVEN SYSTEM}

\section{A. General aspects}

To derive quantum Langevin equation from a microscopic picture we consider the well-known standard systemreservoir model with following form of Hamiltonian ${ }^{31}$

$$
\hat{H}=\frac{\hat{p}^{2}}{2 m}+V(\hat{x}, t)+\sum_{j=1}^{N}\left\{\frac{\hat{p}_{j}^{2}}{2}+\frac{1}{2} \kappa_{j}\left(\hat{q}_{j}-\hat{x}\right)^{2}\right\}
$$

* e-mail address: pcdsr@mahendra.iacs.res.in 
Here $\hat{x}$ and $\hat{p}$ are the coordinate and momentum operators of the particle and $\left\{\hat{q}_{j}, \hat{p}_{j}\right\}$ are the set of coordinate and momentum operators for the reservoir oscillators coupled linearly through the coupling constants $\kappa_{j}(j=1,2, \ldots)$. The potential $V(\hat{x}, t)$ is due to the external force field for the Brownian particle. The coordinate and momentum operators follow the usual commutation rules $\{\hat{x}, \hat{p}\}=i \hbar$ and $\left\{\hat{q}_{i}, \hat{p}_{j}\right\}=i \hbar \delta_{i j}$. Eliminating the bath degrees of freedom in the usual way we obtain the operator Langevin equation for the particle

$$
m \ddot{\hat{x}}+\int_{0}^{t} d t^{\prime} \gamma\left(t-t^{\prime}\right) \dot{\hat{x}}\left(t^{\prime}\right)+V^{\prime}(\hat{x}, t)=\hat{F}(t)
$$

where the noise operator $\hat{F}(t)$ and the memory kernel $\gamma(t)$ are given by

$$
\hat{F}(t)=\sum_{j}\left[\left\{\hat{q}_{j}(0)-\hat{x}(0)\right\} \kappa_{j} \cos \omega_{j} t+\kappa_{j}^{1 / 2} \hat{p}_{j}(0) \sin \omega_{j} t\right]
$$

and

$$
\gamma(t)=\sum_{j} \kappa_{j} \cos \omega_{j} t
$$

respectively, with $\kappa_{j}=\omega_{j}^{2}$. Very recently it has been shown[28-30] that on the basis of quantum mechanical average $\langle\ldots\rangle$ over the bath modes with coherent states and the system with an arbitrary state Eq.(2.2) can be cast into the form of the generalized quantum Langevin equation.

$$
m \ddot{x}+\int_{0}^{t} d t^{\prime} \gamma\left(t-t^{\prime}\right) \dot{x}\left(t^{\prime}\right)+V^{\prime}(x, t)=f(t)+Q\left(x,\left\langle\delta \hat{x}^{n}\right\rangle\right)
$$

where the quantum mechanical mean value of the position operator $\langle\hat{x}\rangle=x$. Here the quantum dispersion term $Q$ to the potential, is given by

$$
Q\left(x,\left\langle\delta \hat{x}^{n}\right\rangle\right)=V^{\prime}(x, t)-\left\langle V^{\prime}(\hat{x}, t)\right\rangle
$$

which by expressing $\hat{x}(t)=x(t)+\delta \hat{x}(t)$ in $V(\hat{x}, t)$ and using a Taylor series expansion around $x$ may be rewritten as

$$
Q\left(x,\left\langle\delta \hat{x}^{n}\right\rangle\right)=-\sum_{n \geq 2} \frac{1}{n !} V^{n+1}(x, t)\left\langle\delta \hat{x}^{n}\right\rangle
$$

The calculation of $Q$ rests on the quantum correction terms $\left\langle\delta \hat{x}^{n}\right\rangle$ which one determines by solving a set of quantum correction equations(as discussed in the later part of this section). Furthermore the c-number Langevin force is given by

$$
f(t)=\sum_{j}\left[\left\{\left\langle\hat{q}_{j}(0)\right\rangle-\langle\hat{x}(0)\rangle\right\} \kappa_{j} \cos \omega_{j} t+\kappa_{j}^{1 / 2} \hat{p}_{j}(0) \sin \omega_{j} t\right]
$$

which must satisfy noise characteristics of the bath at equilibrium ,

$$
\begin{aligned}
\langle f(t)\rangle_{S} & =0 \\
\left\langle f(t) f\left(t^{\prime}\right)\right\rangle_{S} & =\frac{1}{2} \sum_{j} \kappa_{j} \hbar \omega_{j}\left(\operatorname{coth} \frac{\hbar \omega_{j}}{2 k T}\right) \cos \omega_{j}\left(t-t^{\prime}\right)
\end{aligned}
$$


Eq.110) expresses the quantum fluctuation-dissipation relation. The above conditions (2.9-2.10) can be fulfilled provided the initial shifted co-ordinates $\left\{\left\langle\hat{q}_{j}(0)\right\rangle-\langle\hat{x}(0)\rangle\right\}$ and momenta $\left\langle\hat{p}_{j}(0)\right\rangle$ of the bath oscillators are distributed according to a canonical thermal Wigner distribution ${ }^{32.33}$ of the form

$$
P_{j}\left(\left[\left\langle\hat{q}_{j}(0)\right\rangle-\langle\hat{x}(0)\rangle\right],\left\langle\hat{p}_{j}(0)\right\rangle\right)=N \exp \left\{-\frac{\frac{1}{2}\left\langle\hat{p}_{j}(0)\right\rangle^{2}+\frac{1}{2} \kappa_{j}\left[\left\langle\hat{q}_{j}(0)\right\rangle-\langle\hat{x}(0)\rangle\right]^{2}}{\hbar \omega_{j}\left[\bar{n}\left(\omega_{j}\right)+\frac{1}{2}\right]}\right\}
$$

so that the statistical averages $\langle\ldots\rangle_{s}$ over the quantum mechanical mean value $O$ of the bath variables are defined as

$$
\left\langle O_{j}\right\rangle_{s}=\int O_{j} P_{j} d\left\langle\hat{p}_{j}(0)\right\rangle d\left\{\left\langle\hat{q}_{j}(0)\right\rangle-\langle\hat{x}(0)\rangle\right\}
$$

Here $\bar{n}(\omega)$ is given by Bose-Einstein distributions $\left(e^{\frac{\hbar \omega}{k T}}-1\right)^{-1} . P_{j}$ is the exact solution of Wigner equation for harmonic oscillator ${ }^{32.33}$ and forms the basis for description of the quantum noise characteristics of the bath kept in thermal equilibrium at temperature $T . N$ is the normalization constant. In the continuum limit the fluctuationdissipation relation (10) can be written as

$$
\left\langle f(t) f\left(t^{\prime}\right)\right\rangle_{s}=\frac{1}{2} \int_{0}^{\infty} d \omega \kappa(\omega) \rho(\omega) \hbar \omega \operatorname{coth}\left(\frac{\hbar \omega}{2 k T}\right) \cos \omega\left(t-t^{\prime}\right)
$$

where we have introduced the density of the modes $\rho(\omega)$. Since we are interested in the Markovian limit in the present context, we assume $\kappa(\omega) \rho(\omega)=\frac{2}{\pi} \gamma$, and Eq.(13) then yields ${ }^{34}$

$$
\left\langle f(t) f\left(t^{\prime}\right)\right\rangle_{s}=2 D_{q} \delta\left(t-t^{\prime}\right)
$$

with

$$
D_{q}=\frac{1}{2} \gamma \hbar \omega_{0} \operatorname{coth} \frac{\hbar \omega_{0}}{2 k T}
$$

$\omega_{0}$ refers to static frequency limit. Furthermore from Eq.(4) in the continuum limit we have

$$
\gamma\left(t-t^{\prime}\right)=\gamma \delta\left(t-t^{\prime}\right)
$$

$\gamma$ is the dissipation constant in the Markovian limit. In this limit Eq.(5) therefore reduces to

$$
m \ddot{x}+\gamma \dot{x}+V^{\prime}(x, t)=f(t)+Q\left(x,\left\langle\delta \hat{x}^{n}\right\rangle\right)
$$

In order to consider the stochastic dynamics in a bistable system with fluctuating barrier height we consider the potential as,

$$
V(x, t)=-\left[\frac{a}{2}+\frac{\xi(t)}{2}\right] x^{2}+\frac{b}{4} x^{4}
$$

$\xi(t)$ is the external fluctuations with zero mean and is characterized by the following equations

$$
\overline{\xi(t)}=0 ; \quad \overline{\xi(t) \xi\left(t^{\prime}\right)}=2 Q_{I} \delta\left(t-t^{\prime}\right)
$$

where $Q_{I}$ is the strength of noise. Care must be taken to distinguish between the quantum mechanical mean $\langle\hat{O}\rangle(=O)$, the statistical average over the quantum mechanical mean $\langle O\rangle_{s}$ denoting thermal bath average and the statistical average over the external noise $\bar{O}$.

Attention is restricted here to overdamped Langevin equation in one variable $x$ so that we write

$$
\gamma \dot{x}=\left(a x-b x^{3}\right)+x \xi(t)+f(t)+Q\left(x,\left\langle\delta \hat{x}^{n}\right\rangle\right)
$$


$Q\left(x,\left\langle\delta \hat{x}^{n}\right\rangle\right)$ being the correction term due to nonlinearity of the potential is too small to destroy bistablity or the symmetry of the system. If we now apply an weak periodic forcing to the particle, the double-well potential is tilted asymmetrically up and down and the corresponding stochastic differential equation reads as

$$
\gamma \dot{x}=\left(a x-b x^{3}\right)+x \xi(t)+f(t)+Q\left(x,\left\langle\delta \hat{x}^{n}\right\rangle\right)+A \cos \omega t
$$

The Fokker-Planck equation corresponding to Eq. (21) is given by

$$
\frac{\partial P(x, t)}{\partial t}=\frac{\partial}{\partial x}\left[B(x, t)+\frac{\partial}{\partial x} D(x)\right] P(x, t)
$$

where

$$
B(x, t)=-\frac{1}{\gamma}\left[\left(a x-b x^{3}\right)+\frac{x Q_{I}}{\gamma}-Q\left(x,\left\langle\delta \hat{x}^{n}\right\rangle\right)+A \cos \omega t\right]
$$

and,

$$
D(x)=\frac{1}{\gamma^{2}}\left[Q_{I} x^{2}+D_{q}\right]
$$

Eq.(22) can then be rearranged into the following form

$$
\frac{\partial P(x, t)}{\partial t}=\frac{1}{\gamma} \frac{\partial}{\partial x}\left[-a x+b x^{3}-\frac{Q_{I} x}{\gamma}-A \cos \omega t-Q\left(x,\left\langle\delta \hat{x}^{n}\right\rangle\right)+\frac{1}{\gamma} \frac{\partial}{\partial x}\left(D_{q}+Q_{I} x^{2}\right)\right] P(x, t)
$$

The quantum nature of the stochastic dynamics is manifested in two terms; the quantum correction to the classical potential $Q$ and the quantum diffusion coefficient $D_{q}$ characterizing the thermal bath. Eq.(25) is the quantum counterpart of Eq.(5) derived in Ref ${ }^{24}$. Furthermore Eq.(25) formally takes care of quantum correction to all orders and the vacuum field effect of the bath at zero temperature.

\section{B. Static tilting}

In the presence of static tilting, i.e., $\omega=0$, the stationary solution of the Fokker-Planck equation reads as

$$
P_{0}(x, A)=N_{0}\left[x^{2}+\frac{D_{q}}{Q_{I}}\right]^{-\frac{1}{2}+\gamma k\left(1+\frac{k D_{q}}{2 \Delta V}\right)} G(x) \exp \left[-\gamma\left(\frac{k x^{2}}{x_{0}^{2}}-\frac{A}{|x|}\right)\right]
$$

The term $G(x)$, arises due to quantum corrections to the potential and is given by the following expression

$$
G(x)=\exp \left[-\int_{0}^{x} d x^{\prime} \frac{\gamma Q\left(x^{\prime},\left\langle\delta \hat{x}^{n}\right\rangle\right)}{Q_{I}\left(x^{\prime 2}+\frac{D_{q}}{Q_{I}}\right)}\right]
$$

$N_{0}$ is a suitable normalization constant, $k=\frac{a}{2 Q_{I}} ; \Delta V$ and $\pm x_{0}$ denote the barrier height and stable minima of the bistable potential $-\frac{a}{2} x^{2}+\frac{b}{4} x^{4}$, respectively, given by the following expressions

$$
\Delta V=\frac{a^{2}}{4 b} ; \quad x_{0}=\left(\frac{a}{b}\right)^{\frac{1}{2}}
$$

To proceed further it is necessary to find out the quantum correction term $Q\left(x,\left\langle\delta \hat{x}^{n}\right\rangle\right)$ more explicitly. From Eq. (7) we have

$$
Q\left(x,\left\langle\delta \hat{x}^{n}\right\rangle\right)=-\frac{V^{\prime \prime \prime}(x, t)}{2 !}\left\langle\delta \hat{x}^{2}\right\rangle-\frac{V^{\prime \prime \prime \prime}(x, t)}{3 !}\left\langle\delta \hat{x}^{3}\right\rangle+\ldots
$$

For a bistable potential $V^{n+1}(x, t)=0$ for $n \geq 4$. For a minimum uncertainty state $\left\langle\delta \hat{x}^{2}\right\rangle=O(\hbar)$ and therefore the higher order terms of the order $\left\langle\delta \hat{x}^{3}\right\rangle$ can be safely neglected. Furthermore since the stochastic system is overdamped 
the quantum corrections are primarily relevant in the time scale $O\left(\frac{1}{\gamma}\right)$ It is therefore important to calculate the leading order quantum correction $-\frac{V^{\prime \prime \prime}(x, t)}{2}\left\langle\delta \hat{x}^{2}\right\rangle$. To this end we consider the overdamped version of operator equation (2) and use $\hat{x}(t)=x(t)+\delta \hat{x}(t)$, where $x(t)=\langle\hat{x}(t)\rangle$ is the quantum mechanical mean value of the operator $\hat{x}$. By construction $[\delta \hat{x}, \delta \hat{p}]=i \hbar$ and $\langle\delta \hat{x}\rangle=0$. We then make use of the overdamped version Eq.(17) and obtain the operator equation after quantum mechanical average over bath modes with product separable coherent states

$$
\gamma \delta \dot{\hat{x}}+V^{\prime \prime}(x, t) \delta \hat{x}+\sum_{n \geq 2} \frac{1}{n !} V^{n+1}(x, t)\left(\delta \hat{x}^{n}-\left\langle\delta \hat{x}^{n}\right\rangle\right)=0
$$

With the help of operator equation (30) we obtain the coupled equations for $\left\langle\delta \hat{x}^{n}\right\rangle$

$$
\begin{gathered}
\frac{d}{d t}\left\langle\delta \hat{x}^{2}\right\rangle=-\frac{1}{\gamma}\left[2 V^{\prime \prime}(x, t)\left\langle\delta \hat{x}^{2}\right\rangle+V^{\prime \prime \prime}(x, t)\left\langle\delta \hat{x}^{3}\right\rangle\right] \\
\frac{d}{d t}\left\langle\delta \hat{x}^{3}\right\rangle=-\frac{1}{\gamma}\left[3 V^{\prime \prime}(x, t)\left\langle\delta \hat{x}^{3}\right\rangle+\frac{3}{2} V^{\prime \prime \prime}(x, t)\left\langle\delta \hat{x}^{4}\right\rangle-\frac{3}{2} V^{\prime \prime \prime}(x, t)\left\langle\delta \hat{x}^{2}\right\rangle^{2}\right]
\end{gathered}
$$

and so on. To take into account of the leading order contribution $\left\langle\delta \hat{x}^{2}\right\rangle$ to $Q\left(x,\left\langle\delta \hat{x}^{n}\right\rangle\right)$ explicitly we retain only the first term in Eq. (31)

$$
d\left\langle\delta \hat{x}^{2}\right\rangle=-\frac{2}{\gamma} V^{\prime \prime}(x, t)\left\langle\delta \hat{x}^{2}\right\rangle d t
$$

The overdamped deterministic motion on the other hand gives $\gamma d x=-V^{\prime}(x, t) d t$ which can be used in Eq.(33) to eliminate $d t$ and obtain after integration

$$
\int_{\left\langle\delta \hat{x}^{2}\right\rangle_{c}}^{\left\langle\delta \hat{x}^{2}\right\rangle} \frac{d\left\langle\delta \hat{x}^{2}\right\rangle}{\left\langle\delta \hat{x}^{2}\right\rangle}=2 \int_{x_{c}}^{x} \frac{V^{\prime \prime}(x, t)}{V^{\prime}(x, t)}
$$

where $x_{c}$ is a quantum mechanical mean position at which $\left\langle\delta \hat{x}^{2}\right\rangle$ becomes minimum, i. e., $\left\langle\delta \hat{x}^{2}\right\rangle_{x_{c}}=\frac{1}{2} \hbar / \omega_{0}, w_{0}$ being defined in Eq.(15). Explicit integration yields

$$
\left\langle\delta \hat{x}^{2}\right\rangle=\Delta_{c}\left[V^{\prime}(x, t)\right]^{2}
$$

where $\Delta_{c}$ is given by $\Delta_{c}=\frac{\left\langle\delta \hat{x}^{2}\right\rangle_{x_{c}}}{\left[V^{\prime}\left(x_{c}, t\right)\right]^{2}}$. This quantum correction parameter can be estimated as follows:

From Eq.(18) we have

$$
\left[V^{\prime}(x, t)\right]^{2}=x^{2}\left[a^{2}+\xi^{2}(t)+2 a \xi(t)+2(a+\xi(t)) b x+b^{2} x^{4}\right]
$$

By averaging Eq.(36) over external noise $\xi(t)$ using Eq.(19) we have from Eq. (35)

$$
\left\langle\delta \hat{x}^{2}\right\rangle=\Delta_{c} x^{2}\left(a^{2}+Q_{I}+b^{2} x^{4}-2 a b x^{2}\right)
$$

The minimum of $\left\langle\delta \hat{x}^{2}\right\rangle$ at $x=x_{c}$ can be obtained from Eq. (37) as $x_{c}=\left[\frac{a^{2}+Q_{I}}{4 a b}\right]^{1 / 2}$ by neglecting $b^{2}$ term (since $a \gg b)$.

With the help of Eq. 35) it is easy to obtain from Eq. (29) the leading order quantum correction averaged over external noise as given by (we have dropped the overbar sign used in Eq.(19), for convenance)

$$
Q\left(x,\left\langle\delta \hat{x}^{n}\right\rangle\right)=-6 \Delta_{q} b x^{3}\left(a^{2}+Q_{I}+b^{2} x^{4}-2 a b x^{2}\right)
$$

where $\Delta_{q}=\frac{\Delta_{c}}{2} . Q\left(x,\left\langle\delta \hat{x}^{n}\right\rangle\right)$ in Eq.(38) is an odd function, which contributes to the potential force term, so that the system retains its symmetry (i.e., reflection symmetry of the bistable potential remain unchanged)

Now we are in position to discuss the properties of the stochastic processes $x(t)$, in terms of its steady state distribution function $P(x, A)$ for different regimes of coherent and inherent system parameters. 
In absence of the static tilting i.e., $A=0$, the process $x(t)$ diffuses on the entire $x$ axis, the relevant distribution function is symmetric for $x \rightarrow-x$ and $\langle x(t)\rangle=0$. As temperature of the system decreases the probability distribution decreases at the origin, since $D_{q}$ decreases with temperature. In the classical limit as $T \rightarrow 0, D_{q} \rightarrow \gamma k_{B} T \rightarrow 0$ we observe $P(x, 0) \rightarrow 0$ at the origin. This is shown in solid line in Fig.1. So the process $x(t)$ remains confined in any one half axis depending on the initial condition. Unlike the classical limit at $T=0$ the distribution function $P(x, 0)$ in the quantum limit takes the following form;

$$
P_{0}(x, 0)=N_{0}\left[x^{2}+\frac{D_{q}(0)}{Q_{I}}\right]^{-\frac{1}{2}+k\left(1+\frac{k D_{q}(0)}{2 \Delta V}\right)} \exp \left[-\frac{k x^{2}}{x_{0}^{2}}\left(1-\Delta_{1}\right)\right]
$$

Here $\gamma$ is assumed to be unity, $D_{q}(0)$ is zero point diffusion coefficient having non-zero value $\frac{1}{2} \hbar \omega_{0}, \Delta_{1}$ is the contribution due to system nonlinearity correction as given by

$$
\Delta_{1}=6 \Delta_{q}\left(a^{2}+Q_{I}\right)
$$

Hence the process $x(t)$ does not remain confined in the one half axis depending on the initial condition even at zero temperature due to quantum mechanical tunneling. This is an important aspect of bath quantization. The distribution function peaks at $x_{m} \sim \pm\left(\frac{n x_{0}^{2}}{k\left(1-\Delta_{1}\right)}-\frac{D_{q}(0)}{Q_{I}}\right)^{\frac{1}{2}}$, where $n=-\frac{1}{2}+k\left(1+\frac{k D_{q}(0)}{2 \Delta V}\right)$. Thus compared to classical limit the peaks of the distribution function shift slightly towards the origin due to zero point contribution of the bath oscillators. All the above observations are in the limit $a>Q_{I}$ and are shown in Fig.1 and Fig.2. In the zero temperature limit as the external noise intensity is increased to $Q_{I}=a$, the distribution function Eq.(39) attains an unimodal form since $\frac{D_{q}(0)}{\Delta V} \leq 1$; a phenomenon like phase transition occurs both in the classical and the quantum limit. This is presented in the Fig.3. In the quantum case the distribution function deviates from the Gaussian shape due to quantum corrections arising out of higher order nonlinearity. It is important to note that the quantum particle is more localized than the classical particle over the $x$-axis, a feature typical of quantum localization as shown in this case. For $Q_{I}>a$, the distribution function Eq.(39) tends to be singular at the origin since $n<0$ and the appearance of long tails is characterized by stochastic stabilization.

\section{Periodic tilting}

When the periodic tilting is present $\omega \neq 0$ and $A \neq 0$, the process $x(t)$ is no-longer stationary and the timedependent distribution function $P(x, A, t)$ is necessary to describe its steady-state. However in the limit of low forcing frequency ${ }^{24}$ there is enough time for the system to reach the local equilibrium during the period of $\frac{1}{\omega}$. Then the quasi-steady state distribution function reads as

$$
P(x)=N G(x)\left[x^{2}+\frac{D_{q}}{Q_{I}}\right]^{-\frac{1}{2}+k\left(1+\frac{k D_{q}}{2 \Delta V}\right)} \exp \left[-\frac{k x^{2}}{x_{0}^{2}}-L(x)\right]
$$

where the time $\tau$ is used as fixed parameter so that

$$
L(x)=\frac{A \cos \omega \tau}{\left(D_{q} Q_{I}\right)^{\frac{1}{2}}} \arctan \left[\left(\frac{Q_{I}}{D_{q}}\right)^{\frac{1}{2}} x\right]
$$

The quasi-steady state distribution function $P(x)$ is plotted in Fig.4 and Fig.5 as a function of position coordinate when the strength of additive and multiplicative noises $D_{q}$ and $Q_{I}$, respectively, are varied. The probability density function $P(x)$ is depicted in Fig.4 for several values of quantum diffusion constant and for a fixed value of $A(=-0.1)$. The peak located at $x=-\left(\frac{a}{b}\right)^{\frac{1}{2}}$ is much higher than that at $x=+\left(\frac{a}{b}\right)^{\frac{1}{2}}$ when $D_{q}=0.9$. When $D_{q}$ is increased to 10 , the distribution function $P(x)$ gets broadened and the left peak is decreased to almost equal height to the right one. Fig.5 depicts a situation where the multiplicative noise strength is varied. It is observed that the peak of the distribution function at $x=-\left(\frac{a}{b}\right)^{\frac{1}{2}}$ is decreased and moves towards the origin when the strength of the external noise is increased from 0.08 to 0.7 with a gradual lose of Gaussian form.

\section{THE CO-OPERATIVE EFFECT; MULTIPLICATIVE STOCHASTIC RESONANCE}

In the previous section we have been analyzed the stochastic process $x(t)$ in terms of the probability distribution functions. We have compared the distribution function of quantum mechanical mean values with the corresponding 
classical one in the different parameter regime which reflect the profound effect of bath quantization at very low temperature. But this is not sufficient to describe completely the cooperative effect among the inherent thermal noise, the external noise and weak periodic signal. To analyze such nonlinear cooperative effect, i.e., the phenomenon of stochastic resonance in the bistable system the most common way to quantify the effect is through signal-to-noise ratio (SNR). SNR is defined as the ratio of the strength of $\delta$-spike of the power spectrum to the back ground noise level. Following ${ }^{5.7}$ the expression of SNR in the bistable system can be derived from the well-known two-state model approach as

$$
S N R=\frac{\pi}{2} W_{0}\left(\frac{A x_{0}}{D_{q}}\right)^{2}\left(1-\frac{1}{2}\left(\frac{A x_{0}}{D_{q}}\right)^{2}\left[\frac{4 W_{0}^{2}}{4 W_{0}^{2}+\omega^{2}}\right]\right)^{-1}
$$

where $W_{0}$ is the Kramer's hopping rate over the activated barrier in absence of periodic force and $\pm x_{0}$ are the two stable states.

The simplest way to calculate Kramer's rate is through mean first passage time (MFPT). The exact expression for $\mathrm{MPFT}^{27}$ for a particle to reach the final point $x_{2}$, the quantum mechanical mean position from an initial point $x_{1}$ is given by $\left(W_{0}=\frac{1}{T_{m}}\right)$

$$
T_{m}\left(x_{1} \rightarrow x_{2}\right)=\int_{x_{1}}^{x_{2}} \frac{d x}{D(x) P_{s}(x)} \int_{-\infty}^{x} P_{s}(x) d y
$$

From Eq.(22]- 24), we obtain the stationary distribution $P(x)(A=0$ and $\gamma=1)$ as

$$
P_{s}(x)=N D^{-\frac{1}{2}}(x) \exp \left[\frac{-\bar{U}(x)}{Q_{I}}-\int_{0}^{x} \frac{Q\left(x,\left\langle\delta \hat{x}^{n}\right\rangle\right)}{D(x)} d x\right]
$$

where

$$
D(x)=Q_{I} x^{2}+D_{q} ; \quad \bar{U}(x)=-\int_{0}^{x} \frac{a y-b y^{3}}{\left(y^{2}+\frac{D_{q}}{Q_{I}}\right) d y}
$$

The two points $x_{1}$ and $x_{2}$ are $-x_{0}$ and 0 , respectively. Now using steepest-descent approximation we obtain the expression for $T_{m}$ in the usual way

$$
T_{m}\left(x_{1} \rightarrow x_{2}\right)=2 \pi\left|U^{\prime \prime}\left(x_{1}\right) U^{\prime \prime}\left(x_{2}\right)\right|^{-\frac{1}{2}} \exp \left[\frac{\bar{U}\left(x_{1}\right)-\bar{U}\left(x_{2}\right)}{Q_{I}}\right] \exp \left[\phi\left(x_{2}\right)-\phi\left(x_{1}\right)\right]
$$

where

$$
\phi(x)=\int_{0}^{x} \frac{Q\left(y,\left\langle\delta \hat{y}^{n}\right\rangle\right)}{D(y)} d y ; \quad U(x)=-\frac{a}{2} x^{2}+\frac{b}{4} x^{4}
$$

Having determined the hopping rate we are in position to explain the effect of different parameters on the signal-tonoise ratio. From the equation the effect of increasing input signal $A$ is quite straightforward. On increasing the input signal one observes the increase of SNR. The implication of varying the signal frequency is, however, complicated. To explore this effect we present in Fig.6 the variation of signal-to-noise ratio as function of temperature for several values of signal frequency $(\omega)$. The solid curve represents the value of SNR, for lowest frequency $(\omega=0.0001)$ and dashed refers to the same for the largest frequency $(\omega=0.1)$. The SNR decreases as the frequency is increased in the lower temperature range. When the signal frequency is low, the particle gets time to approach a local equilibrium, and it has a high likelihood of hopping from upper well to the lower well during the half cycle. Hence a type of resonance phenomenon is expected to occur at optimum level of thermal fluctuation. At higher temperature such type of nonlinear cooperative effect is destroyed due to substantial probability of hopping back to the upper well. At the high frequency regime on the other hand such nonlinear cooperative effect is less likely to be observed, because a fewer number of particles find the time to hop to the lower well during each cycle. On increasing temperature one may increase the inter-well transition rate, but at the risk of being kicked in the anti-phase direction. Although SNR passes through a maximum as one varies the thermal noise strength it may be considered as some type of compromise between input signal and inherent thermal fluctuation rather than a resonance.

Fig.7 depicts the variation of SNR as a function of temperature comparing the classical and the quantum limits and shows that SNR is larger in quantum limit at low temperature and the difference become insignificant at high temperature as expected. It is also interesting to note that in contrast to classical limit SNR is not zero at absolute zero due to zero point contribution of the thermal bath. 


\section{CONCLUSION}

Based on the study of stationary probability distribution function and signal-to-noise ratio we have investigated the problem of quantum stochastic dynamics of a system in a bistable fluctuating potential which is driven by a slow periodic or static field. Our conclusions are summarized as follows:

(i) Temperature profoundly affects the characteristic of the bimodal stationary probability distribution function which reveals that in contrast to classical case, where the system remains confined in one of the wells, quantization allows a finite population at barrier top even at zero due to vacuum field effect of the thermal bath in absence of tilting and for a low noise strength of the modulation for fluctuating well. Thus although at zero temperature the passage between the wells is classically forbidden, quantum tunneling makes the passage smooth for population transfer.

(ii) For a static tilting and a higher strength of stochastic modulation of the well, the unimodal stationary probability distribution becomes more localized and acquires non-Gaussian character on quantization as compared to that for the corresponding classical system.

(iii) Very slow external periodic driving may localize the system in one of the wells. The peak of the distribution function shifts towards the origin as the strength of modulation of fluctuating potential is increased.

(iv) The effect of quantization also makes its presence felt significantly in the signal-to-noise ratio at low temperature.

The method presented here takes care of the statistical properties of the thermal bath in terms of Wigner canonical thermal distribution which remains a valid pure state distribution even at absolute zero. This help us to consider the vacuum field limit in the treatment of stochastic processes the governing equation of which is classical in form but quantum mechanical in its content. Moreover although formally one takes into account of the quantum correction terms due to nonlinearity of the potential to all orders in the stationary distribution function, it is possible to estimate these corrections order by order for actual calculation effectively to a good degree of accuracy. We thus hope that our observations will be useful for experimental studies on tailored quantum systems like quantum dots at very low temperature.

\section{Acknowledgments}

Thanks are due to the Council of Scientific and Industrial Research, Government of India, for a fellowship (PKG).

1 A. Schenzle and H. Brand, Phys. Rev. A, 20, 1628 (1979)

2 B. Lindiner, J. Garcia-ojalvo, A. Neiman, L. Schimansky-Geier, Phys. Reps. 392321 (2004)

3 J. Garcia-ojalvo and J. M. Sancho, noise in spatially extened systems (springer-verlag, NY, 1999)

4 R. Benzi, G. Parisi, A. Sutera, and A. Vulpiani, Tellus 34, 10 (1982); R. Benzi, A. Sutera, and A. Vulpiani, J. Phys. A 14 L 453 (1981)

5 B. McNamara and K. Wiesenfeld, Phys. Rev. A, 39, 4854 (1988); L. Gammaitoni, F. Marchesoni, E. Menichella-Saetta and S. Santucci, Phys. Rev. Lett. 62, 349 (1989).

${ }^{6}$ B. McNamara, K. Wiesenfeld and R. Roy, Phys. Rev. Lett. 60, 2628 (1988).

7 L. Gammaitoni, P. Hänggi, P. Jung and F.Marchesoni, Rev. Mod. Phys., 70, 223(1998)

8 L. Q. Zhou, X. Jia and Q. Ouyang, Phys. Rev. Lett. 88, 138301(2002)

9 J. Garcia-Ojalvo, A. Hernadez-Machado and J. M. Sancho, Phys. Rev. Lett. 71, 1542 (1993)

10 A. Becker and L. Kramer, Phys. Rev. Lett. 73, 955 (1994), A. Becker and L. Kramer, Physica D 90, 408(1995)

11 J. M. R. Parrondo, C. Van den Broeck, J. Buceta and F. J. de la Rubia, Physica A 224, 153(1996)

12 A. A. Zaikin and L. Schimansky-Geier, Phys. Rev. E. 58, 4355(1998)

13 J. Buceta, M. Ibanes, J. M. Sancho and K. Lindenberg, Phys. Rev. E67, 021113(2003)

14 S. Dutta, S. S. Riaz and D. S. Ray, Phys. Rev. E 71, 036216(2005)

15 W. Horsthemke and R. Lefever, Noise Induced Transition, Theory and Application in Physics, Chemistry and Biology, (Springer, Berlin 1983)

16 A. S. Pikovsky and J. Kurths, Phys. Rev. Lett. 78, 775 (1997).

17 P. Reiman, Phys. Reps. 361, 57 (2002).

18 F. Julicher, A. Ajdari and J. Prost, Rev. Mod. Phys. 69, 1269 (1997).

19 M. O. Magnasco, Phys. Rev. Lett. 71, 1477 (1993).

${ }^{20}$ H. Linke, W. Sheng, A. Löfgren, H. Xu, P. Omling and P. E. Lindelof, Europhys. Lett., 44, 341 (1998)

21 H. Schanz, M. Otto, R. Ketzmerick and T. Dittrich, Phys. Rev. Lett. 87, 070601 (2001)

22 T. H. Humphrcy, H. Linke and R. Ncwbury, Physica E 11, 281 (2001)

23 R. Lfstedt and S. N. Coppersmith, Phys. Rev. Lett. 72, 1947 (1993); J. Juhi-Lian Ting, Phys. Rev. E 59, 2801 (1999)

${ }^{24}$ L. Gammaitoni, F. Marchesoni, E. Menichella -Saetta, and S. Santucci Phys. Rev. E 49, 4878 (1993) 
25 X. Luo and S. Zhu, Phys. Rev. E 67, 021104 (2003)

26 Sergey M. Bezrukov and Igor Vodyanoy, Nature 385, 319 (1997); V. Narayan and S. Stafström, Phys. Rev. B 69, 75315 (2004)

27 R. L. Straonovich, Topics in the Theory of Random Noise, Vol. 1 (Gordon and Breach, New York, 1963); K. Lindenberg and B.J. West, J. Stat. Phys. 42, 201 (1986); J. Masoliver, B.J. West, and K. Lindenberg, Phys. Rev. A 35, 3086 (1987); D. Mei, G. Xie, Li Cao, and D. Wu, Phys. Rev. E 59, 3880 (1999)

28 D. Banerjee, B. C. Bag, S. K. Banik and D. S. Ray, Phys. Rev. E 65, 021109 (2002).

29 D. Banerjee, B. C. Bag, S. K. Banik and D. S. Ray, J. Chem. Phys. 120, 8960 (2004).

30 D. Barik and D. S. Ray, J. Chem. Phys. 121, 1681 (2004).

31 R. Zwanzig, J. Stat. Phys.9, 215 (1973).

32 E. P. Wigner, Phys. Rev. 40, 749 (1932).

${ }^{33}$ M. Hillery, R. F. O'Connell, M. O. Scully and E. P. Wigner, Phys. Reps. 106, 121 (1984).

${ }^{34}$ W. H. Louisell, Quantum Statistical Properties of Radiation (J. Wiley, 1973) 


\section{Figure Captions}

Fig.1: Variation of probability density function $(P(x))$ as a function of quantum mechanical mean position $(\mathrm{x})$ in absence of tilting $(\mathrm{A}=0)$ for the parameter set $a=1.0, b=0.06, \Delta_{q}=0.02, Q_{I}=0.1$ and (i) $D_{q}=0.0\left(\gamma k_{B} T=0\right)$ and $\Delta_{q}=0.0$ (solid line) [classical] (ii) $D_{q}=0.5$ (dashed line), (iii) $D_{q}=3.0$ (dotted line) and (iv) $D_{q}=5.00$ (dash-dot line).

Fig.2: Variation of $\mathrm{P}(\mathrm{x})$ as a function of $\mathrm{x}$ at zero temperature comparing quantum(b) and classical(a) limits for the parameter set $a=1.0, b=0.006, Q_{I}=0.5, A=0.0$ and $\Delta_{q}=0.02$.

Fig.3: Variation of $\mathrm{P}(\mathrm{x})$ as a function of $\mathrm{x}$ at zero temperature limit comparing quantum(b) and classical(a) phase transition for the parameter set $a=1.0, b=0.006, A=0.0, \Delta_{q}=0.02$ and (i) $a>Q_{I}=0.5$ (dotted line) and $Q_{I}=a=1.0$ (solid line)

Fig.4: Variation of $P(x)$ with $x$ for different values of quantum quantum diffusion coefficient $\left(D_{q}\right), D_{q}=0.9$ (solid line), $D_{q}=3.5$ (dotted line) and $D_{q}=10.0$ (dashed line) in presence of tilting (A=-0.1) for $a=1.0, b=0.006$, $Q_{I}=0.1, \omega=0.1, \Delta_{q}=0.02$ and $\tau=1$

Fig.5: Variation of $P(x)$ with $x$ for different values of external multiplicative noise strength $\left(Q_{I}\right), Q_{=} 0.05$ (solid line), $Q_{I}=0.1$ (dotted line), $Q_{I}=0.3$ (dashed line) and $Q_{I}=0.7$ (dash-dot line) in presence of tilting $(\mathrm{A}=-0.1)$ for $a=1.0, b=0.006, Q_{I}=0.1, \omega=0.1, \Delta_{q}=0.02$ and $\tau=1$

Fig.6: Variation of Signal-to-noise(SNR) ratio with temperature(T) for the parameter set $a=2.5, b=0.001$, $Q_{I}=3.0, \Delta_{q}=0.02, A=0.008$ and (i) $\omega=0.0001$ (solid line), (ii) $\omega=0.001$ (dotted line) and (iii) $\omega=0.1$ (dashed line)

Fig.7: A plot of signal-to-noise ratio vs temperature comparing quantum and classical limit for the parameter set $a=2.5, b=0.001, Q_{I}=3.0, \Delta_{q}=0.01, A=0.008$ and $\omega=0.1$ (i) classical case (solid line), (ii) quantum case (dotted line) 


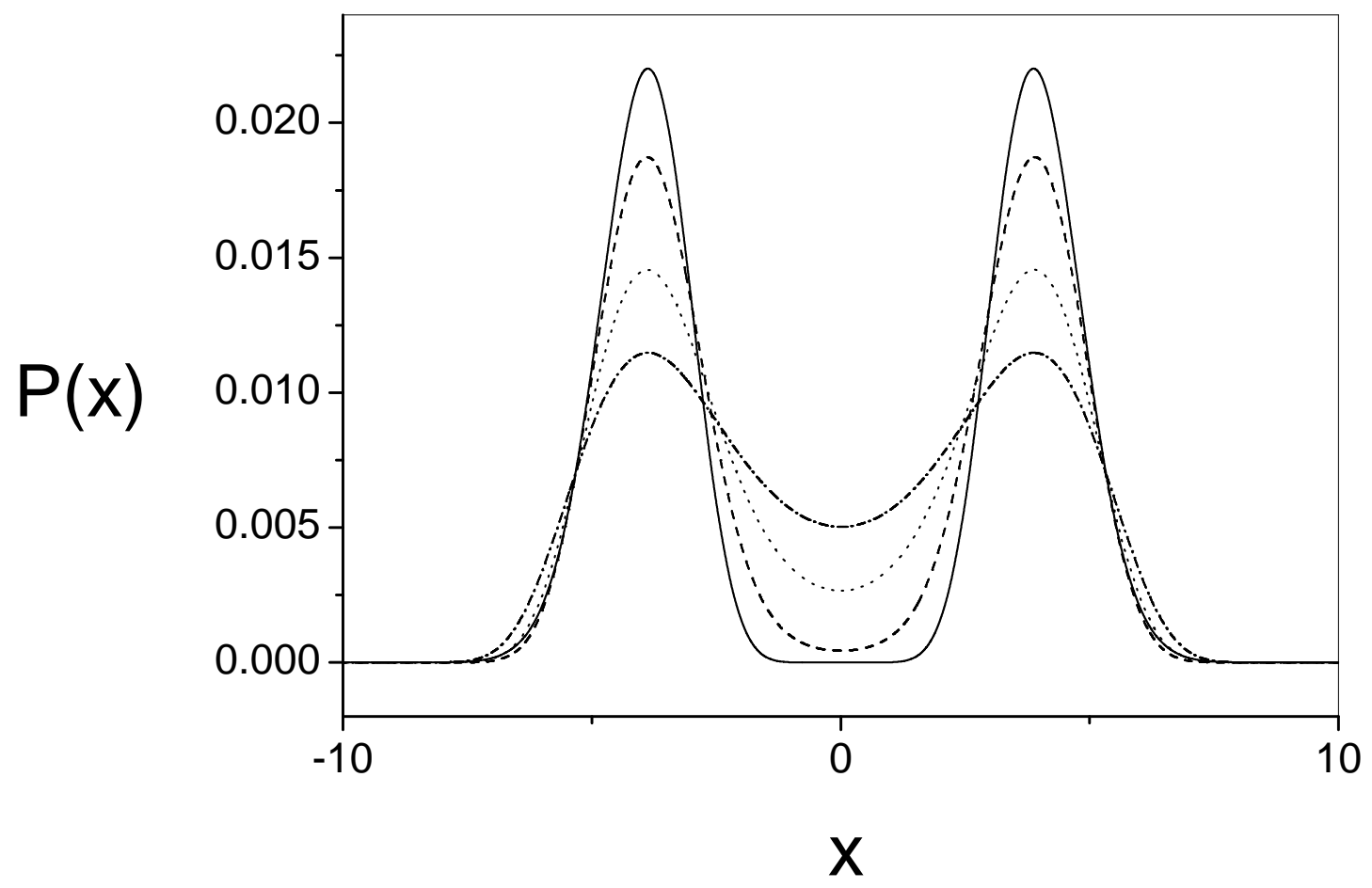

Fig.1 

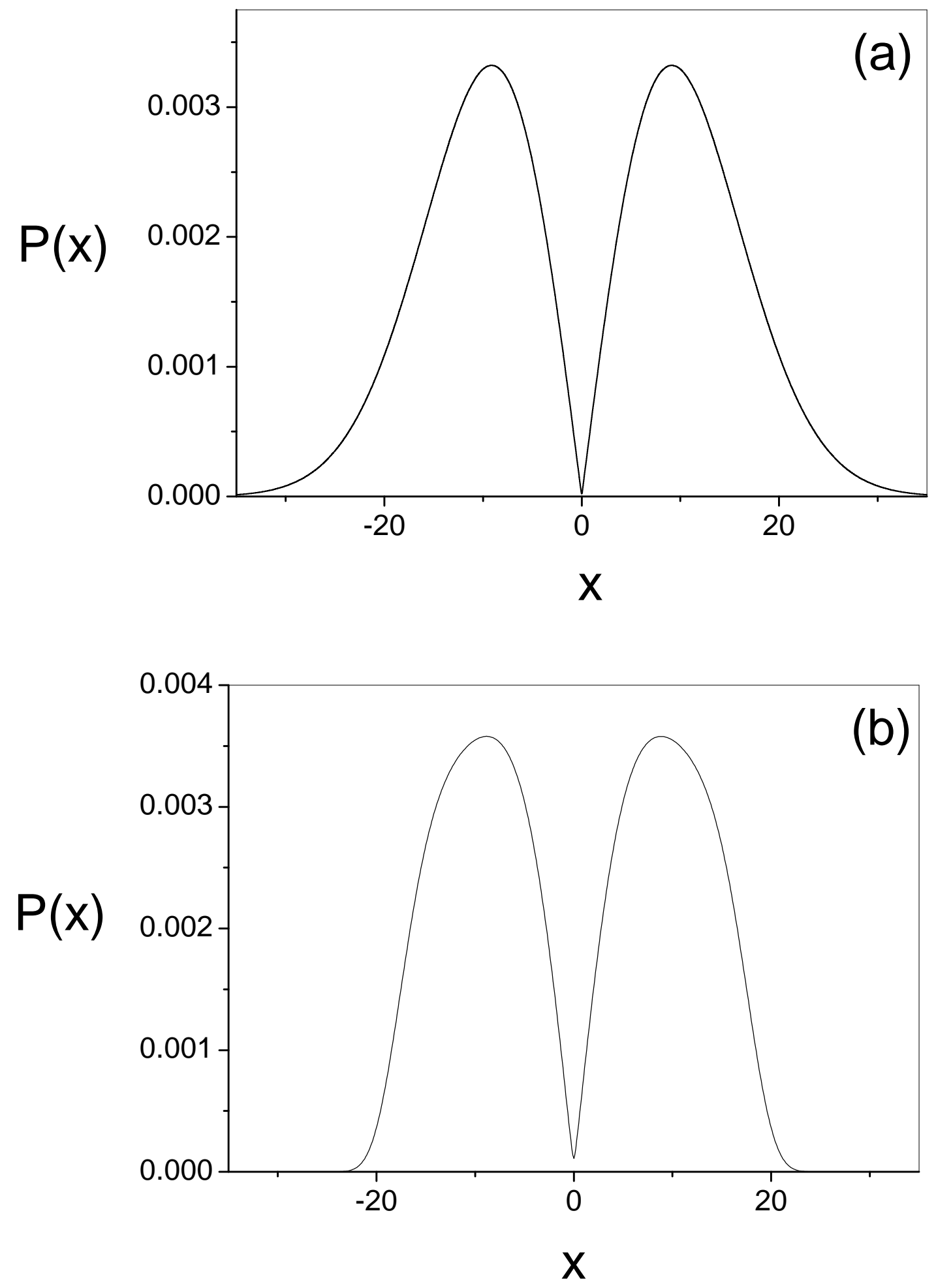

Fig.2 

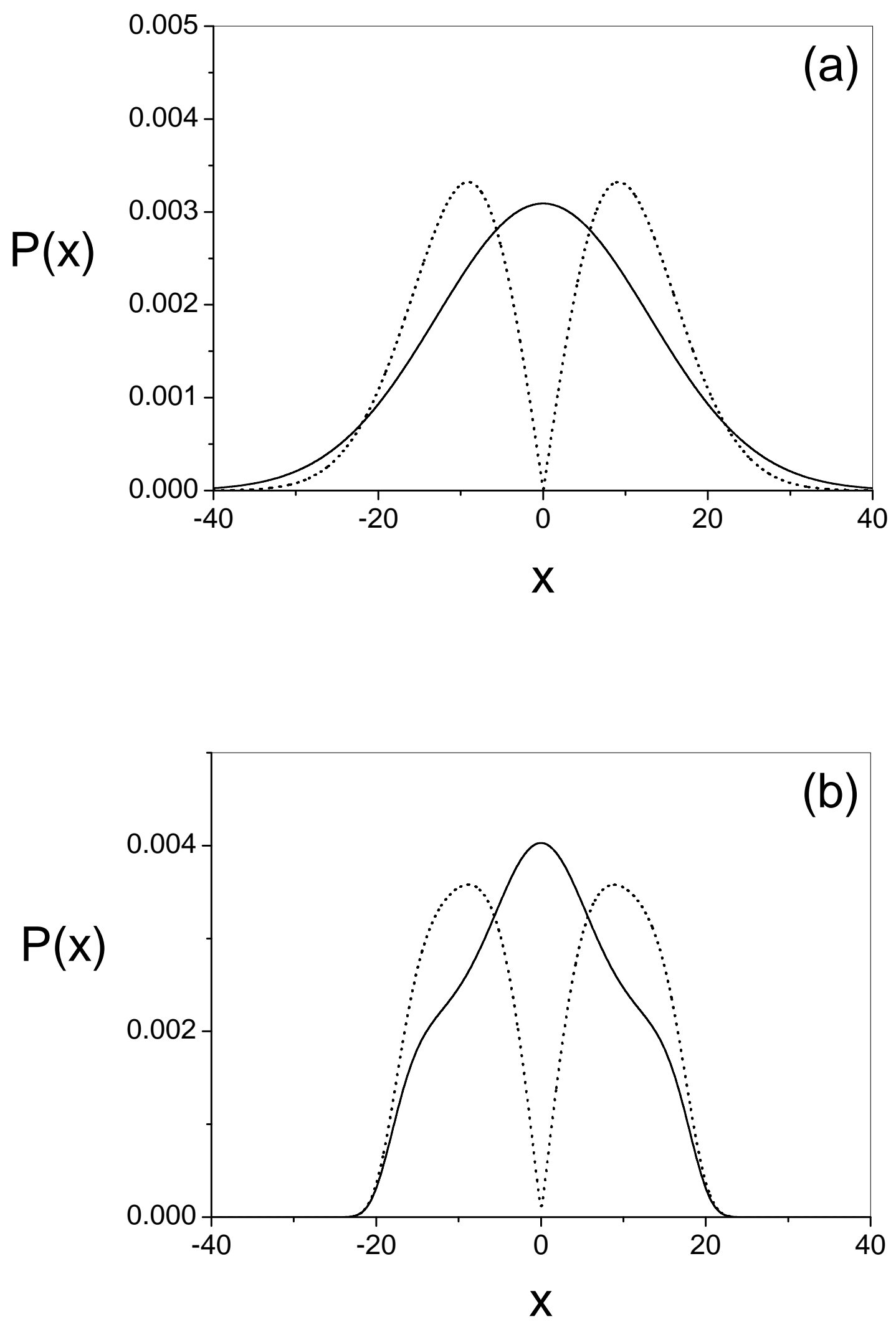

Fig.3 


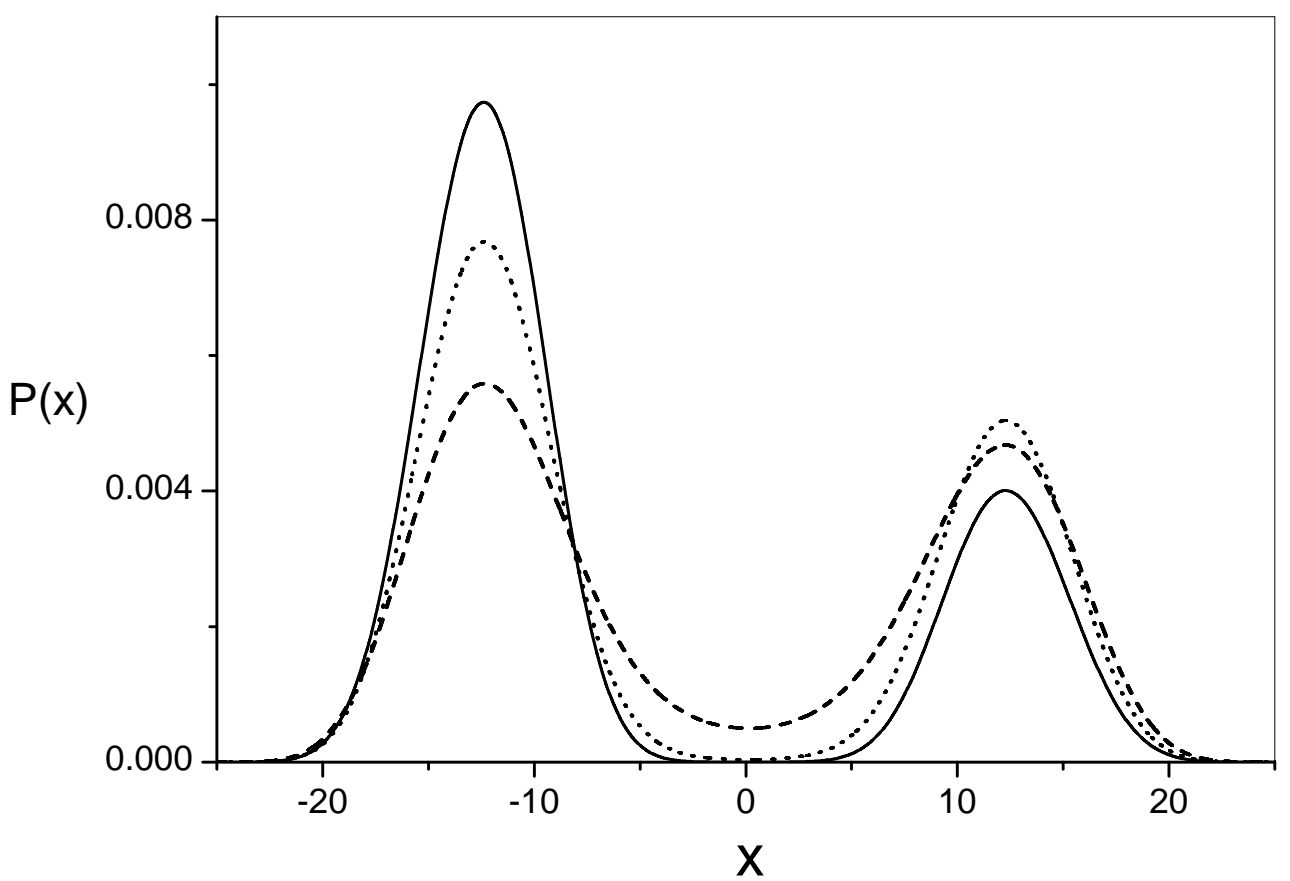

Fig.4 


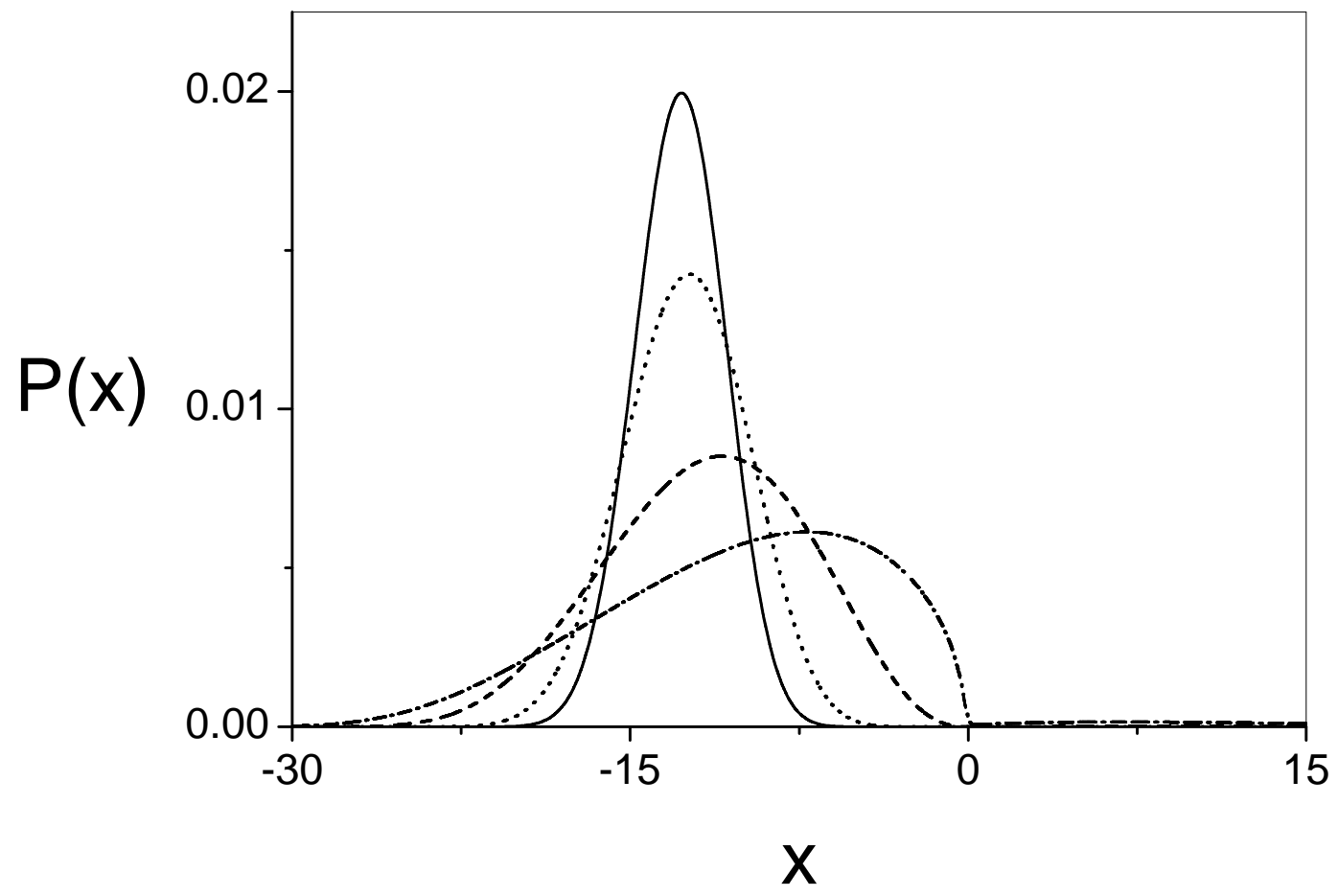

Fig.5 


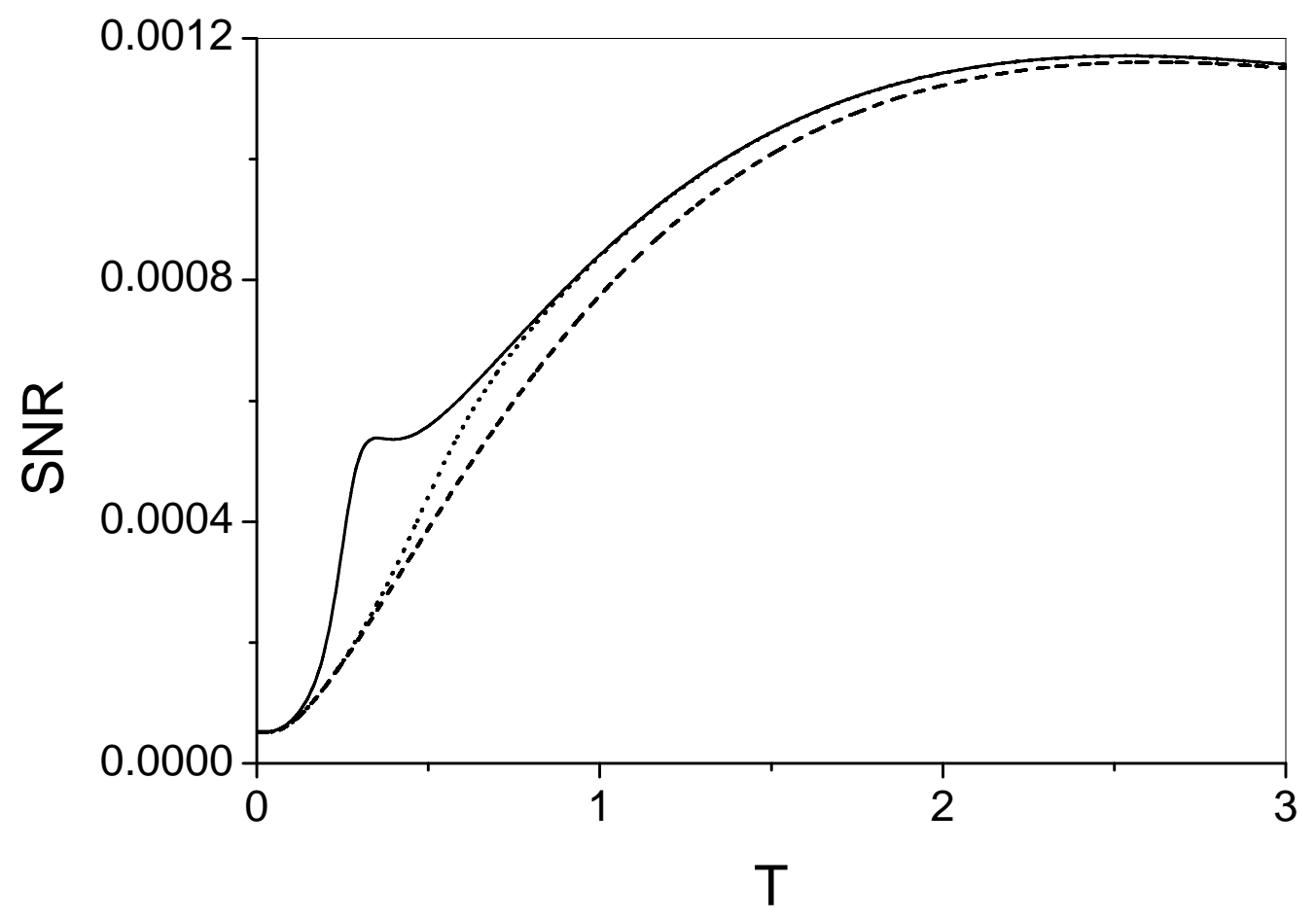

Fig.6 


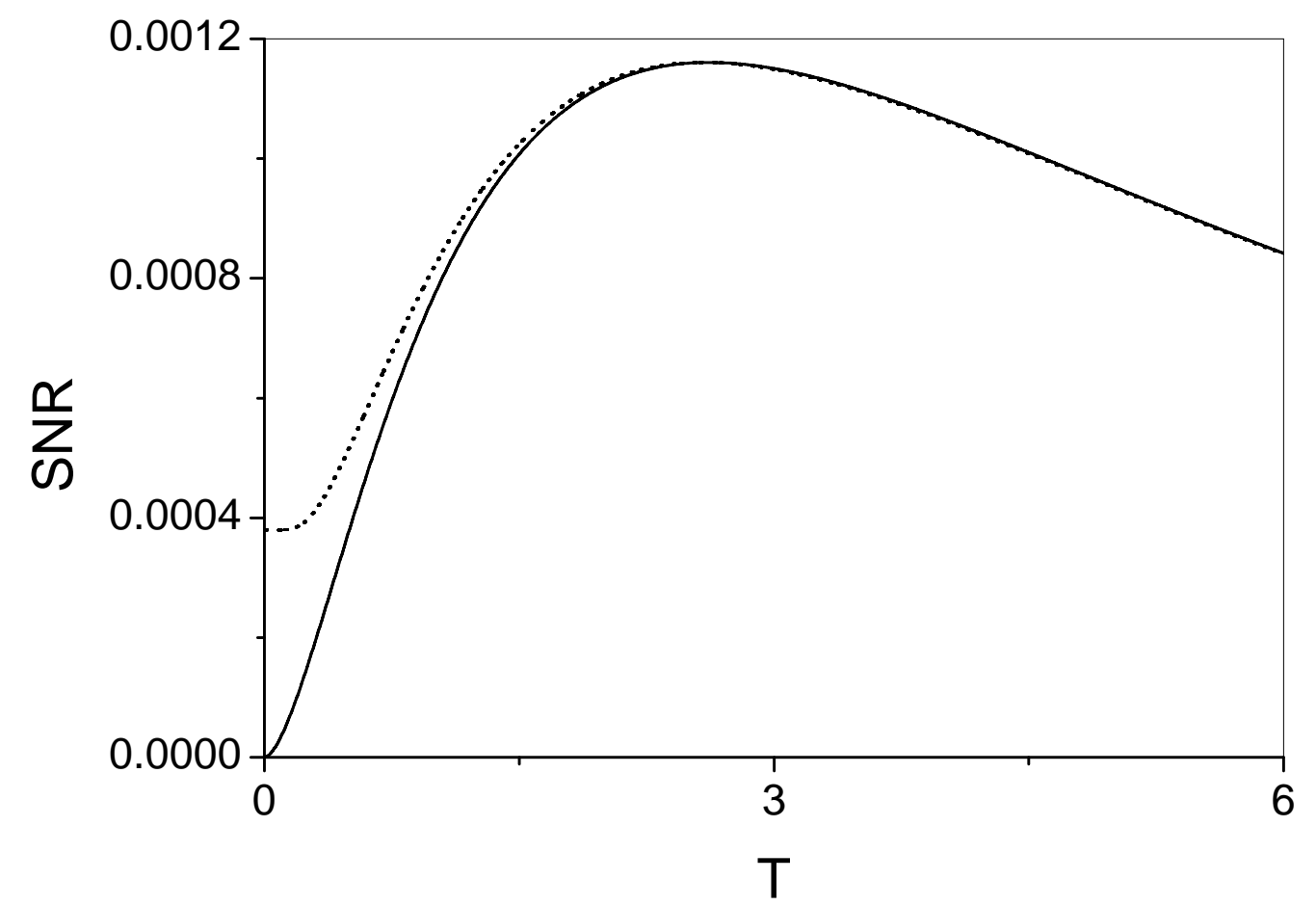

Fig.7 